EPJ Web of Conferences 71, 00011 (2014)

DOI: 10.1051/epjconf/20147100011

(C) Owned by the authors, published by EDP Sciences, 2014

\title{
New results and perspectives of DAMA/LIBRA
}

\author{
R. Bernabei ${ }^{1,2}$, P. Belli ${ }^{2}$, F. Cappella ${ }^{3,4}$, V. Caracciolo ${ }^{5}$, S. Castellano ${ }^{5}$, R. Cerulli ${ }^{5}$, C.J. Dai ${ }^{6}$, \\ A. d'Angelo ${ }^{3,4}$, S. d'Angelo ${ }^{1,2}$, A. Di Marco ${ }^{1,2}$, H.L. He ${ }^{6}$, A. Incicchitti ${ }^{4}$, H.H.Kuang ${ }^{6}$, X.H. Ma ${ }^{6}$, \\ F. Montecchia ${ }^{2,7}$, X.D. Sheng ${ }^{6}$, R.G. Wang ${ }^{6}$, and Z.P. Ye $\mathrm{e}^{6,8}$ \\ ${ }^{1}$ Dip. di Fisica, Università di Roma "Tor Vergata", I-00133 Rome, Italy \\ 2 INFN, sez. Roma "Tor Vergata", I-00133 Rome, Italy \\ ${ }^{3}$ Dip. di Fisica, Università di Roma "La Sapienza", I-00185 Rome, Italy \\ ${ }^{4}$ INFN, sez. Roma, I-00185 Rome, Italy \\ ${ }^{5}$ INFN, Laboratori Nazionali del Gran Sasso, Assergi, Italy \\ ${ }^{6}$ IHEP, Chinese Academy, P.O. Box 918/3, Beijing 100039, China \\ ${ }^{7}$ Dip. di Ingegneria Civile e Ingegneria Informatica, Università di Roma "Tor Vergata" \\ ${ }^{8}$ University of Jing Gangshan, Jiangxi, China
}

\begin{abstract}
The results obtained with the total exposure of 1.04 ton $\times \mathrm{yr}$ collected by DAMA/LIBRA-phase1 deep underground at the Gran Sasso National Laboratory (LNGS) of the I.N.F.N. during 7 annual cycles are briefly summarized. The DAMA/LIBRA-phase1 and the former DAMA/NaI data (cumulative exposure 1.33 ton $\times$ yr, corresponding to 14 annual cycles) give evidence at $9.3 \sigma$ C.L. for the presence of Dark Matter (DM) particles in the galactic halo, on the basis of the exploited model independent $\mathrm{DM}$ annual modulation signature by using highly radio-pure $\mathrm{NaI}(\mathrm{Tl})$ target. The modulation parameters are well in agreement with those expected for DM particles. No systematic or side reaction able to mimic the exploited DM signature has been found or suggested by anyone over more than a decade. Some of the perspectives of the presently running DAMA/LIBRA-phase2 are outlined.
\end{abstract}

\section{Introduction}

The presently running DAMA/LIBRA [1-11] experiment, as the former DAMA/NaI [12-25], has the main aim to investigate the presence of DM particles in the galactic halo by exploiting the model independent DM annual modulation signature (originally suggested in Ref. [26]). Moreover, the developed highly radio-pure $\mathrm{NaI}(\mathrm{Tl})$ target-detectors [1] assure as well sensitivity to a wide range of DM candidates, interaction types and astrophysical scenarios.

As a consequence of the Earth's revolution around the Sun, the Earth should be crossed by a larger flux of DM particles around $\simeq 2$ June and by a smaller one around $\simeq 2$ December. This DM annual modulation signature is very distinctive since the effect induced by DM particles must simultaneously satisfy all the following requirements: the rate must contain a component modulated according to a cosine function (1) with one year period (2) and a phase that peaks roughly $\simeq 2$ June (3); this modulation must only be found in a well-defined low energy range, where DM particle induced events can be present (4); it must apply only to those events in which just one detector of many actually "fires"

This is an Open Access article distributed under the terms of the Creative Commons Attribution License 2.0, which permits unrestricted use, distribution, and reproduction in any medium, provided the original work is properly cited. 
(single-hit events), since the DM particle multi-interaction probability is negligible (5); the modulation amplitude in the region of maximal sensitivity must be $\simeq 7 \%$ for usually adopted halo distributions (6), but it can be larger in case of some possible scenarios such as e.g. those in Ref. [27, 28] (even up to $\simeq 30 \%$ ). Thus this signature is model independent, very effective and, in addition, it allows the test of a large range of cross sections and of halo densities.

The full description of the DAMA/LIBRA set-up during the phase 1 and other related arguments have been discussed in details in Ref. $[1-4,8]$ and references therein. Here we just remind that the sensitive part of this set-up is made of 25 highly radiopure $\mathrm{NaI}(\mathrm{Tl})$ crystal scintillators (5-rows by 5 -columns matrix) having $9.70 \mathrm{~kg}$ mass each one. In each detector two $10 \mathrm{~cm}$ long UV light guides (made of Suprasil B quartz) act also as optical windows on the two end faces of the crystal, and are coupled to two low background photomultipliers (PMTs) working in coincidence at single photoelectron level. The low background 9265-B53/FL and 9302-A/FL PMTs, developed by EMIElectron Tubes with dedicated R\&Ds, were used in the phase1; for details see Ref. [1, 14, 16] and references therein. The detectors are housed in a sealed low-radioactive copper box installed in the center of a low-radioactive $\mathrm{Cu} / \mathrm{Pb} / \mathrm{Cd}$-foils/polyethylene/paraffin shield; moreover, about $1 \mathrm{~m}$ concrete (made from the Gran Sasso rock material) almost fully surrounds (mostly outside the barrack) this passive shield, acting as a further neutron moderator. A threefold-levels sealing system prevents the detectors to be in contact with the environmental air of the underground laboratory [1]. The light response of the detectors during phase1 typically ranges from 5.5 to 7.5 photoelectrons $/ \mathrm{keV}$, depending on the detector. The hardware threshold of each PMT is at single photoelectron, while a software energy threshold of $2 \mathrm{keV}$ electron equivalent (hereafter $\mathrm{keV}$ ) is used [1, 14]. Energy calibration with X-rays $/ \gamma$ sources are regularly carried out in the same running condition down to few $\mathrm{keV}$ [1]; in particular, double coincidences due to internal X-rays from ${ }^{40} \mathrm{~K}$ (which is at ppt levels in the crystals) provide (when summing the data over long periods) a calibration point at $3.2 \mathrm{keV}$ close to the software energy threshold (for details see Ref. [1]). The radiopurity, the procedures and details are discussed in Ref. [1-4, 8] and references therein.

\section{The results of DAMA/LIBRA-phase 1 and DAMA/Nal}

The total exposure of DAMA/LIBRA-phase 1 is: 1.04 ton $\times$ yr in seven annual cycles; when including also that of the first generation DAMA/NaI experiment it is 1.33 ton $\times \mathrm{yr}$, corresponding to 14 annual cycles. For details see Ref. [2-4, 8].

Fig. 1 shows the time behaviour of the experimental residual rates of the single-hit scintillation events in the (2-6) keV energy interval for DAMA/LIBRA-phase1. The residuals of the DAMA/NaI data $(0.29$ ton $\times$ yr) are given in Ref. $[2,8,16,17]$. We remind that these residual rates are calculated from the measured rate of the single-hit events after subtracting the constant part: $\left\langle r_{i j k}-\text { flat }_{j k}\right\rangle_{j k}$. Here $r_{i j k}$ is the rate in the considered $i$-th time interval for the $j$-th detector in the $k$-th energy bin, while flat $_{j k}$ is the rate of the $j$-th detector in the $k$-th energy bin averaged over the cycles. The average is made on all the detectors ( $j$ index) and on all the energy bins ( $k$ index) which constitute the considered energy interval. The weighted mean of the residuals must obviously be zero over one cycle.

The $\chi^{2}$ test excludes the hypothesis of absence of modulation in the data: $\chi^{2} /$ d.o.f. $=83.1 / 50$ and the $\mathrm{P}$-value is $\mathrm{P}=2.2 \times 10^{-3}$ for the (2-6) $\mathrm{keV}$ energy interval. When fitting the single-hit residual rate of DAMA/LIBRA-phase1 (Fig. 1) together with the DAMA/NaI ones, with the function: $A \cos \omega\left(t-t_{0}\right)$, considering a period $T=\frac{2 \pi}{\omega}=1 \mathrm{yr}$ and a phase $t_{0}=152.5$ day (June $\left.2^{\text {nd }}\right)$ as expected by the DM annual modulation signature, the following modulation amplitude is obtained: $A=(0.0110 \pm 0.0012) \mathrm{cpd} / \mathrm{kg} / \mathrm{keV}$ corresponding to $9.2 \sigma$ C.L.. 


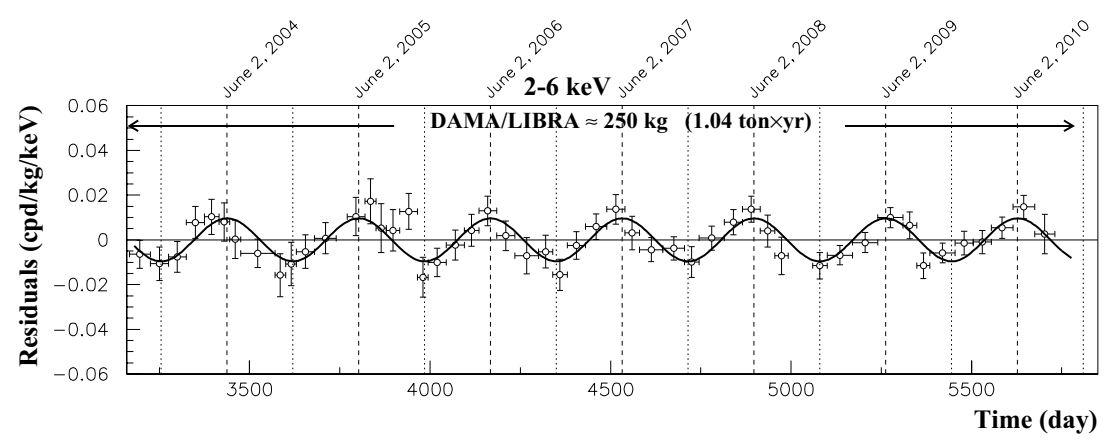

Figure 1. Experimental residual rate of the single-hit scintillation events measured by DAMA/LIBRA-phase1 in the (2-6) keV energy interval as a function of the time. The data points present the experimental errors as vertical bars and the associated time bin width as horizontal bars. The superimposed curves are the cosinusoidal functions behaviours $A \cos \omega\left(t-t_{0}\right)$ with a period $T=\frac{2 \pi}{\omega}=1 \mathrm{yr}$, a phase $t_{0}=152.5$ day (June $2^{\text {nd }}$ ) and modulation amplitudes, $A$, equal to the central values obtained by best fit on the data points of the entire DAMA/LIBRAphase1. The dashed vertical lines correspond to the maximum expected for the DM signal (June $2^{\text {nd }}$ ), while the dotted vertical lines correspond to the minimum.

When the period, and the phase are kept free in the fitting procedure, the modulation amplitude is $(0.0112 \pm 0.0012) \mathrm{cpd} / \mathrm{kg} / \mathrm{keV}(9.3 \sigma$ C.L. $)$, the period $T=(0.998 \pm 0.002)$ year and the phase $t_{0}=(144 \pm 7)$ day. For more details see Ref. [4].

The period and the phase are well compatible with expectations for a DM annual modulation signal. In particular, the phase is consistent with about June $2^{\text {nd }}$ and is fully consistent with the value independently determined by Maximum Likelihood analysis (see later). For completeness, we recall that a slight energy dependence of the phase could be expected in case of possible contributions of non-thermalized DM components to the galactic halo, such as e.g. the SagDEG stream [19, 29, 30] and the caustics [31].

The DAMA/LIBRA-phase1 single-hit residuals of Fig. 1 and those of DAMA/NaI have also been investigated by a Fourier analysis. The data analysis procedure has been described in details in Ref. [8]. A clear peak corresponding to a period of 1 year (see Fig. 2) is evident for the (2-6) keV energy interval; the same analysis in the (6-14) keV energy region shows instead only aliasing peaks. Neither other structure at different frequencies has been observed (see also Ref. [8]).

Absence of any other significant background modulation in the energy spectrum has been verified in energy regions not of interest for $\mathrm{DM}^{1}$; e.g. the measured rate integrated above $90 \mathrm{keV}, \mathrm{R}_{90}$, as a function of the time has been analysed [4].

A further relevant investigation in the DAMA/LIBRA-phase1 data has been performed by applying the same hardware and software procedures, used to acquire and to analyse the single-hit residual rate, to the multiple-hit one. In fact, since the probability that a DM particle interacts in more than one detector is negligible, a DM signal can be present just in the single-hit residual rate. Thus, the comparison of the results of the single-hit events with those of the multiple-hit ones corresponds practically to compare between them the cases of DM particles beam-on and beam-off. This procedure also allows an additional test of the background behaviour in the same energy interval where the pos-

\footnotetext{
${ }^{1}$ In fact, the background in the lowest energy region is essentially due to "Compton" electrons, X-rays and/or Auger electrons, muon induced events, etc., which are strictly correlated with the events in the higher energy region of the spectrum. Thus, if a modulation detected in the lowest energy region were due to a modulation of the background (rather than to a signal), an equal or larger modulation in the higher energy regions should be present.
} 


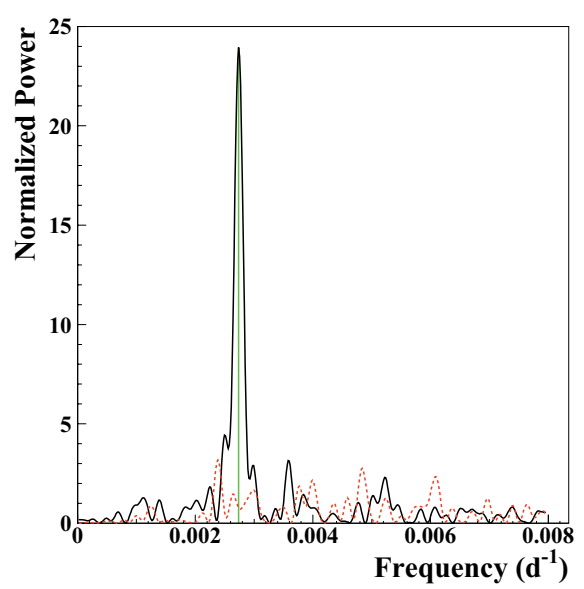

Figure 2. Power spectrum of the measured single-hit residuals in the (2-6) keV (solid lines) and (6-14) keV (dotted lines) energy intervals calculated according to Ref. [8], including also - as usual in DAMA analyses - the treatment of the experimental errors and of the time binning. The data refer to DAMA/NaI and DAMA/LIBRAphase 1 . As it can be seen, the principal mode present in the (2-6) keV energy interval corresponds to a frequency of $2.737 \times 10^{-3} \mathrm{~d}^{-1}$ (vertical lines), corresponding to a period of $\simeq 1$ year. A similar peak is not present in the (6-14) keV energy interval.

itive effect is observed. In particular, in Fig. 3 the residual rates of the single-hit events measured over the DAMA/LIBRA-phase 1 annual cycles are reported, as collected in a single cycle, together with the residual rates of the multiple-hit events, in the (2-6) keV energy interval. While, as already observed, a clear modulation, satisfying all the peculiarities of the DM annual modulation signature, is present in the single-hit events, the fitted modulation amplitude for the multiple-hit residual rate is well compatible with zero: $-(0.0005 \pm 0.0004) \mathrm{cpd} / \mathrm{kg} / \mathrm{keV}$ in the energy region (2-6) $\mathrm{keV}$. Thus, again evidence of annual modulation with proper features as required by the DM annual modulation signature is present in the single-hit residuals (events class to which the DM particle induced events belong), while it is absent in the multiple-hit residual rate (event class to which only background events belong). Similar results were also obtained for the last two annual cycles of the DAMA/NaI experiment [17]. Since the same identical hardware and the same identical software procedures have been used to analyse the two classes of events, the obtained result offers an additional strong support for the presence of a DM particle component in the galactic halo.

The annual modulation present at low energy can also be pointed out by depicting - as a function of the energy - the modulation amplitude, $S_{m}$, obtained by maximum likelihood method.

In Fig. 4 the obtained $S_{m}$ are shown in each considered energy bin (there $\Delta E=0.5 \mathrm{keV}$ ) when the data of DAMA/NaI and DAMA/LIBRA-phase 1 are considered. It can be inferred that positive signal is present in the (2-6) keV energy interval, while $S_{m}$ values compatible with zero are present just above. In fact, the $S_{m}$ values in the (6-20) keV energy interval have random fluctuations around zero with $\chi^{2}$ equal to 35.8 for 28 degrees of freedom (upper tail probability of $15 \%$ ). All this confirms the previous analyses.

As described in Ref. [2-4, 8], it has been verified that the observed annual modulation effect is well distributed in all the 25 detectors at 95\% C.L.

Among further additional tests, the analysis of the modulation amplitudes as a function of the energy separately for the nine inner detectors and the remaining external ones has been carried out for the entire DAMA/LIBRA-phase1. The obtained values are fully in agreement; in fact, the hypothesis 
ICNFP 2013

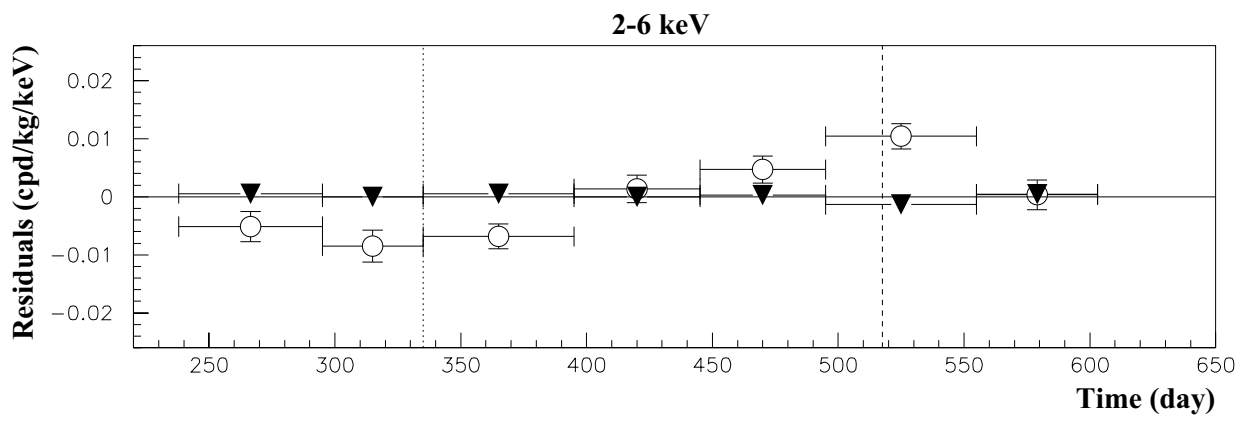

Figure 3. Experimental residual rates of DAMA/LIBRA-phase1 single-hit events (open circles), class of events to which DM events belong, and for multiple-hit events (filled triangles), class of events to which DM events do not belong. They have been obtained by considering for each class of events the data as collected in a single annual cycle and by using in both cases the same identical hardware and the same identical software procedures. The initial time of the figure is taken on August $7^{\text {th }}$. The experimental points present the errors as vertical bars and the associated time bin width as horizontal bars. Analogous results were obtained for the DAMA/NaI data [17].

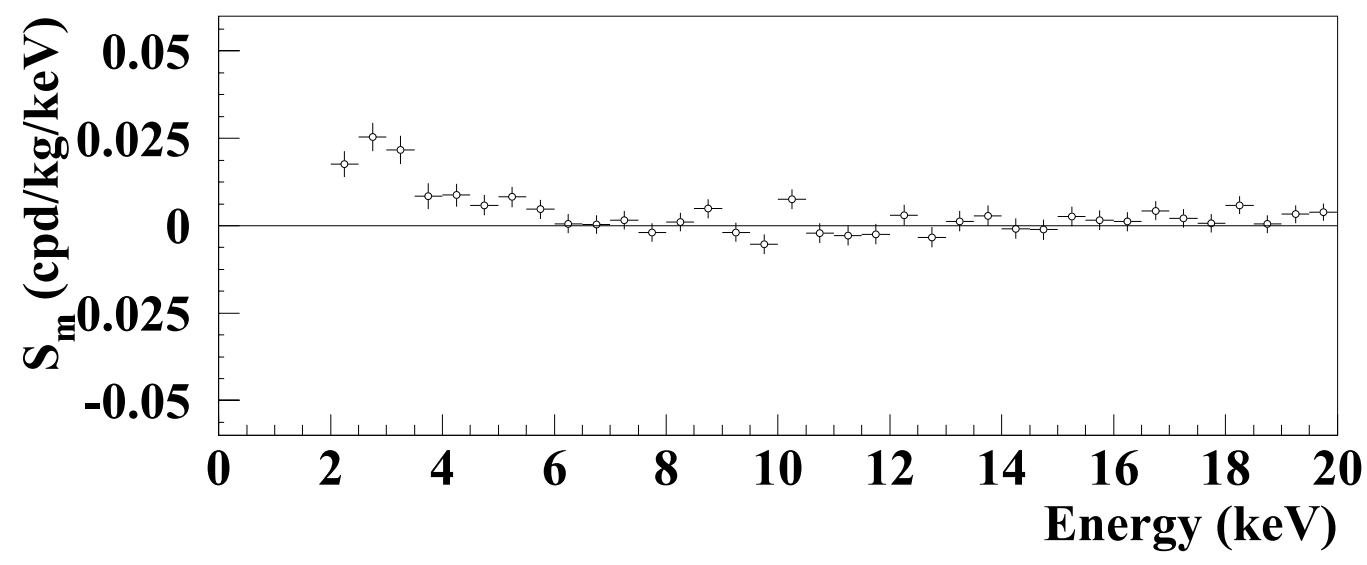

Figure 4. Energy distribution of the $S_{m}$ variable for the total cumulative exposure 1.33 ton $\times$ yr. The energy bin is $0.5 \mathrm{keV}$. A clear modulation is present in the lowest energy region, while $S_{m}$ values compatible with zero are present just above. In fact, the $S_{m}$ values in the (6-20) keV energy interval have random fluctuations around zero with $\chi^{2}$ equal to 35.8 for 28 degrees of freedom (upper tail probability of $15 \%$ ).

that the two sets of modulation amplitudes as a function of the energy belong to same distribution has been verified by $\chi^{2}$ test, obtaining: $\chi^{2} /$ d.o.f. $=3.9 / 4$ and $8.9 / 8$ for the energy intervals (2-4) and (2-6) $\mathrm{keV}$, respectively $(\Delta \mathrm{E}=0.5 \mathrm{keV})$. This shows that the effect is also well shared between inner and outer detectors.

Let us, finally, release the assumption of a phase $t_{0}=152.5$ day in the procedure to evaluate the modulation amplitudes. In this case the signal can be written as:

$$
\begin{aligned}
S_{i k} & =S_{0, k}+S_{m, k} \cos \omega\left(t_{i}-t_{0}\right)+Z_{m, k} \sin \omega\left(t_{i}-t_{0}\right) \\
& =S_{0, k}+Y_{m, k} \cos \omega\left(t_{i}-t^{*}\right) .
\end{aligned}
$$

For signals induced by DM particles one should expect: i) $Z_{m, k} \sim 0$ (because of the orthogonality between the cosine and the sine functions); ii) $S_{m, k} \simeq Y_{m, k}$; iii) $t^{*} \simeq t_{0}=152.5$ day. In fact, these 
conditions hold for most of the dark halo models; however, as mentioned above, slight differences can be expected in case of possible contributions from non-thermalized DM components, such as e.g. the SagDEG stream [19, 29, 30] and the caustics [31].

Considering cumulatively the data of DAMA/NaI and DAMA/LIBRA-phase1 (exposure 1.33 ton $\times$ yr) the obtained $2 \sigma$ contours in the plane $\left(S_{m}, Z_{m}\right)$ for the (2-6) $\mathrm{keV}$ and $(6-14) \mathrm{keV}$ energy intervals are shown in Fig. 5-left while in Fig. 5-right the obtained $2 \sigma$ contours in the plane $\left(Y_{m}, t^{*}\right)$ are depicted. The best fit values $(1 \sigma$ errors $)$ are: $\mathrm{S}_{m}=(0.0106 \pm 0.0012) \mathrm{cpd} / \mathrm{kg} / \mathrm{keV}$,
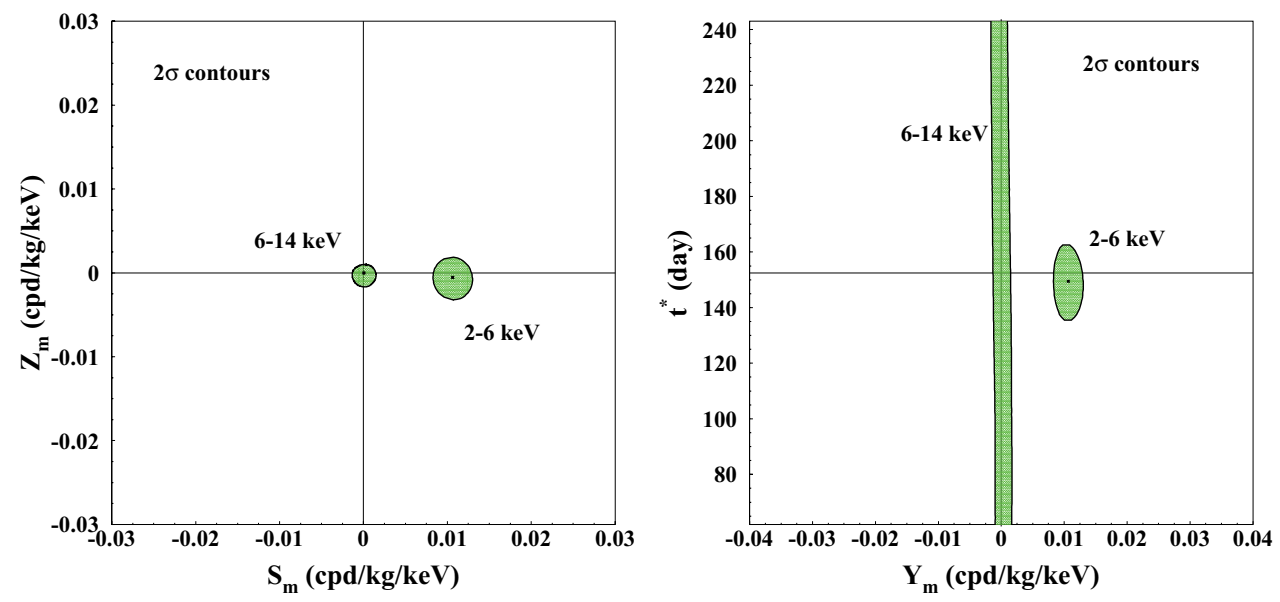

Figure 5. $2 \sigma$ contours in the plane $\left(S_{m}, Z_{m}\right)$ (left) and in the plane $\left(Y_{m}, t^{*}\right)$ (right) for the (2-6) $\mathrm{keV}$ and (614) $\mathrm{keV}$ energy intervals. The contours have been obtained by the maximum likelihood method, considering the cumulative exposure of DAMA/NaI and DAMA/LIBRA-phase1. A modulation amplitude is present in the lower energy intervals and the phase agrees with that expected for DM induced signals. See text.

$\mathrm{Z}_{m}=-(0.0006 \pm 0.0012) \mathrm{cpd} / \mathrm{kg} / \mathrm{keV}, \mathrm{Y}_{m}=(0.0107 \pm 0.0012) \mathrm{cpd} / \mathrm{kg} / \mathrm{keV}$ and $t^{*}=(149.5 \pm 7.0)$ day for the (2-6) $\mathrm{keV}$ energy interval; $S_{m}=(0.0001 \pm 0.0007) \mathrm{cpd} / \mathrm{kg} / \mathrm{keV}, Z_{m}=(0.0000 \pm 0.0005)$ $\mathrm{cpd} / \mathrm{kg} / \mathrm{keV}, \mathrm{Y}_{m}=(0.0001 \pm 0.0008) \mathrm{cpd} / \mathrm{kg} / \mathrm{keV}$ and $t^{*}$ undefined for the (6-14) $\mathrm{keV}$ energy intervals.

Sometimes naive statements were put forwards as the fact that in nature several phenomena may show some kind of periodicity. It is worth noting that the point is whether they might mimic the annual modulation signature in DAMA/LIBRA (and former DAMA/NaI), i.e. whether they might be not only quantitatively able to account for the observed modulation amplitude but also able to contemporaneously satisfy all the requirements of the DM annual modulation signature. The same is also for side reactions. This has already been deeply investigated in Ref. [1-4] and references therein; additional arguments can be found in Ref. [7, 8, 32-38].

In conclusion, the model-independent DAMA results give evidence (at 9.3 $\sigma$ C.L. over 14 independent annual cycles) for the presence of DM particles in the galactic halo.

In order to perform corollary investigation on the nature of the DM particles, model-dependent analyses are necessary; thus, many theoretical and experimental parameters and models are possible and many hypotheses must also be exploited. In particular, the DAMA model-independent evidence is compatible with a wide set of astrophysical, nuclear and particle physics scenarios as also shown in literature. Moreover, both the negative results and all the possible positive hints, achieved so-far in the field, are largely compatible with the DAMA model-independent DM annual modulation results in many scenarios considering also the existing experimental and theoretical uncertainties; the same holds for indirect approaches. For a discussion see e.g. Ref. [8] and references therein. 


\section{DAMA/LIBRA-phase2 and perspectives}

A first upgrade of the DAMA/LIBRA set-up was performed in September 2008. One detector was recovered by replacing a broken PMT and a new optimization of some PMTs and HVs was done; the transient digitizers were replaced with new ones (the U1063A Acqiris 8-bit 1GS/s DC270 High-Speed cPCI Digitizers) having better performances and a new DAQ with optical read-out was installed. The DAMA/LIBRA-phase1 concluded its data taking in this configuration on 2010; the data of the last (seventh) annual cycle of this phase1 will be released soon.

A further and more important upgrade has been performed at the end of 2010 when all the PMTs have been replaced with new ones having higher Quantum Efficiency (Q.E.), realized with a special dedicated development by HAMAMATSU co.. Details on the developments and on the reached performances in the operative conditions are reported in Ref. [6]. Just as example, Fig. 6 shows the values of the Q.E. of the new 50 HAMAMATSU PMTs installed in DAMA/LIBRA both at peak and at the $\lambda$ of the $\mathrm{NaI}(\mathrm{Tl})$ scintillation light. We remind that up to October 2010 low background PMTs,

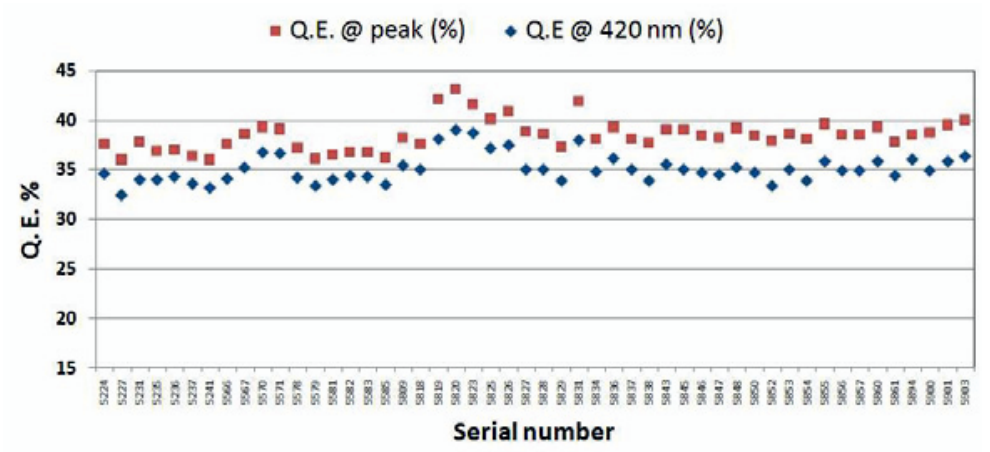

Figure 6. Q.E. at peak and at $420 \mathrm{~nm}$ of each one of the 50 high Q.E. PMTs, installed in DAMA/LIBRA-phase2. The averages (RMS) are 38.5\% (1.6\%) and 35.1\% (1.4\%), respectively; the RMS show that the Q.E. spread in the PMTs production is well limited.

developed by EMI-Electron Tubes with dedicated R\&D, were used; the light yield and other response features already allowed a software energy threshold of $2 \mathrm{keV}$ in the data analysis. The feasibility to decrease the software energy threshold below $2 \mathrm{keV}$ in the new configuration has been demonstrated [6].

Since the fulfillment of this upgrade, the DAMA/LIBRA-phase 2 is continuously running in order: (1) to increase the experimental sensitivity lowering the software energy threshold of the experiment; (2) to improve the corollary investigation on the nature of the DM particle and related astrophysical, nuclear and particle physics arguments; (3) to investigate other signal features. This requires long and heavy full time dedicated work for reliable collection and analysis of very large exposures, as DAMA collaboration has always done.

Another upgrade at the end of 2012 was successfully concluded: new-concept preamplifiers were installed, with suitable operative and electronic features; in particular, they allow the direct connection of the signal to the relative channel of the Transient Digitizer (TD).

Moreover, further improvements are planned. In particular, new trigger modules have been prepared and ready to be installed. A further simplification of the electronic chain has been proposed and funded; for such purpose a new electronic module, New Linear FiFo (NLF), has been designed. It will allow - among the others - a significant reduction of the number of used NIM slots with definitive advantage. 
In the future DAMA/LIBRA will also continue its study on several other rare processes [9-11] as also the former DAMA/NaI apparatus did [24].

Finally, further improvements to increase the sensitivity of the set-up are under evaluation; in particular, the use of high Q.E. and ultra-low background PMTs directly coupled to the $\mathrm{NaI}(\mathrm{Tl})$ crystals is considered $^{2}$. This possible configuration will allow a further large improvement in the light collection and a further lowering of the software energy threshold. Moreover, efforts towards a possible highly radiopure $\mathrm{NaI}(\mathrm{Tl})$ "general purpose" experiment (DAMA/1ton) having full sensitive mass of 1 ton (we already proposed in 1996 as a general purpose set-up) are continuing in various aspects.

\section{Conclusions}

The data of DAMA/LIBRA-phase1 have further confirmed a peculiar annual modulation of the singlehit events in the (2-6) keV energy region satisfying all the many requirements of the DM annual modulation signature; the cumulative exposure by the former DAMA/NaI and DAMA/LIBRA-phase 1 is 1.33 ton $\times \mathrm{yr}$.

In fact, as required by the DM annual modulation signature: 1) the single-hit events show a clear cosine-like modulation as expected for the DM signal; 2) the measured period is equal to $(0.998 \pm$ 0.002) yr well compatible with the $1 \mathrm{yr}$ period as expected for the DM signal; 3 ) the measured phase $(144 \pm 7)$ days is compatible with the roughly $\simeq 152.5$ days expected for the DM signal; 4) the modulation is present only in the low energy (2-6) keV interval and not in other higher energy regions, consistently with expectation for the DM signal; 5) the modulation is present only in the single-hit events, while it is absent in the multiple-hit ones as expected for the DM signal; 6) the measured modulation amplitude in $\mathrm{NaI}(\mathrm{Tl})$ of the single-hit events in the (2-6) $\mathrm{keV}$ energy interval is: $(0.0112 \pm$ $0.0012) \mathrm{cpd} / \mathrm{kg} / \mathrm{keV}(9.3 \sigma$ C.L.). No systematic or side processes able to simultaneously satisfy all the many peculiarities of the signature and to account for the whole measured modulation amplitude is available.

DAMA/LIBRA is continuously running in its new configuration (named DAMA/LIBRA-phase2) with a lower software energy threshold aiming to improve the knowledge on corollary aspects regarding the signal and on second order effects as discussed e.g. in Ref. [8].

\section{References}

[1] R. Bernabei et al., Nucl. Instr. and Meth. A 592 (2008) 297.

[2] R. Bernabei et al., Eur. Phys. J. C 56 (2008) 333.

[3] R. Bernabei et al., Eur. Phys. J. C 67 (2010) 39.

[4] R. Bernabei et al., Eur. Phys. J. C 73 (2013) 2648.

[5] P. Belli et al., Phys. Rev. D 84 (2011) 055014.

[6] R. Bernabei et al., J. of Instr. 7 (2012) P03009.

[7] R. Bernabei et al., Eur. Phys. J. C 72 (2012) 2064.

[8] R. Bernabei et al., Int. J. of Mod. Phys. A 28 (2013) 1330022.

[9] R. Bernabei et al., Eur. Phys. J. C 62 (2009) 327.

[10] R. Bernabei et al., Eur. Phys. J. C 72 (2012) 1920.

[11] R. Bernabei et al., Eur. Phys. J. A 49 (2013) 64.

[12] P. Belli, R. Bernabei, C. Bacci, A. Incicchitti, R. Marcovaldi, D. Prosperi, DAMA proposal to INFN Scientific Committee II, April $24^{\text {th }} 1990$.

\footnotetext{
${ }^{2}$ However, this would require the disassembling of the detectors since the light guides act at present also as optical windows.
} 
[13] R. Bernabei et al., Phys. Lett. B 389 (1996) 757; R. Bernabei et al., Phys. Lett. B 424 (1998) 195; R. Bernabei et al., Phys. Lett. B 450 (1999) 448; P. Belli et al., Phys. Rev. D 61 (2000) 023512; R. Bernabei et al., Phys. Lett. B 480 (2000) 23; R. Bernabei et al., Phys. Lett. B 509 (2001) 197; R. Bernabei et al., Eur. Phys. J. C 23 (2002) 61; P. Belli et al., Phys. Rev. D 66 (2002) 043503.

[14] R. Bernabei et al., Il Nuovo Cim. A 112 (1999) 545.

[15] R. Bernabei et al., Eur. Phys. J. C 18 (2000) 283.

[16] R. Bernabei el al., La Rivista del Nuovo Cimento 26 n.1 (2003) 1-73.

[17] R. Bernabei et al., Int. J. Mod. Phys. D 13 (2004) 2127.

[18] R. Bernabei et al., Int. J. Mod. Phys. A 21 (2006) 1445.

[19] R. Bernabei et al., Eur. Phys. J. C 47 (2006) 263.

[20] R. Bernabei et al., Int. J. Mod. Phys. A 22 (2007) 3155.

[21] R. Bernabei et al., Eur. Phys. J. C 53 (2008) 205.

[22] R. Bernabei et al., Phys. Rev. D 77 (2008) 023506.

[23] R. Bernabei et al., Mod. Phys. Lett. A 23 (2008) 2125.

[24] R. Bernabei et al., Phys. Lett. B408 (1997) 439; P. Belli et al., Phys. Lett. B460 (1999) 236; R. Bernabei et al., Phys. Rev. Lett. 83 (1999) 4918; P. Belli et al., Phys. Rev. C60 (1999) 065501; R. Bernabei et al., Il Nuovo Cimento A112 (1999) 1541; R. Bernabei et al., Phys. Lett. B 515 (2001) 6; F. Cappella et al., Eur. Phys. J.-direct C14 (2002) 1; R. Bernabei et al., Eur. Phys. J. A 23 (2005) 7; R. Bernabei et al., Eur. Phys. J. A 24 (2005) 51; R. Bernabei et al., Astrop. Phys. 4 (1995) 45.

[25] R. Bernabei, in the volume The Identification of Dark Matter, World Sc. Pub. (1997) 574.

[26] K.A. Drukier et al., Phys. Rev. D 33 (1986) 3495; K. Freese et al., Phys. Rev. D 37 (1988) 3388.

[27] D. Smith and N. Weiner, Phys. Rev. D 64 (2001) 043502; D. Tucker-Smith and N. Weiner, Phys. Rev. D 72 (2005) 063509; D. P. Finkbeiner et al, Phys. Rev. D 80 (2009) 115008.

[28] K. Freese et al., Phys. Rev. D 71 (2005) 043516; K. Freese et al., Phys. Rev. Lett. 92 (2004) 11301.

[29] K. Freese et al., Phys. Rev. D 71 (2005) 043516; New Astr. Rev. 49 (2005) 193; astroph/0310334; astro-ph/0309279.

[30] G. Gelmini, P. Gondolo, Phys. Rev. D 64 (2001) 023504.

[31] F.S. Ling, P. Sikivie and S. Wick, Phys. Rev. D 70 (2004) 123503.

[32] R. Bernabei et al., AIP Conf. Proceed. 1223 (2010) 50 [arXiv:0912.0660].

[33] R. Bernabei et al., J. Phys.: Conf. Ser. 203 (2010) 012040 [arXiv:0912.4200]; http://taup2009.lngs.infn.it/slides/jul3/nozzoli.pdf, talk given by F. Nozzoli.

[34] R. Bernabei et al., in the volume Frontier Objects in Astrophysics and Particle Physics, ed. S.I.F. (Vulcano, 2010), p. 157 [arXiv: 1007.0595].

[35] R. Bernabei et al., Can. J. Phys. 89 (2011) 11.

[36] R. Bernabei et al., Physics Procedia 37 (2012) 1095.

[37] R. Bernabei et al., arXiv:1210.6199.

[38] R. Bernabei et al., arXiv:1211.6346. 